\title{
Mechanisms of gram-positive vancomycin resistance (Review)
}

\author{
SAMY SELIM \\ Department of Clinical Laboratory Sciences, College of Applied Medical Sciences, Jouf University, \\ Sakaka 72341, Kingdom of Saudi Arabia
}

Received October 4, 2021; Accepted November 15, 2021

DOI: $10.3892 /$ br.2021.1490

\begin{abstract}
Vancomycin-resistant bacteria (VRB) are important consideration in medicine and public health as they can cause life-threatening infections that appear to be resistant to therapy and persist in the body after medication. A wide spectrum of antimicrobial resistance characteristics, as well as various environmental and animal settings underlie the evolution of the most prevalent the most prevalent van genes in the VRB genome, indicating significant gene flow. As illnesses caused by VRB have become increasingly complex, several previously effective therapeutic techniques have become ineffective, complicating clinical care further. The focus of this review is the mechanism of vancomycin resistance in Enterococci, Staphylococci and Lactobacilli.
\end{abstract}

\section{Contents}

1. Introduction

2. Mechanisms of vancomycin resistant Enterococci (VRE)

3. Vancomycin resistance in Enterococci is conferred by several genes

4. Mechanisms of resistance to vancomycin in Staphylococci

5. Mechanisms of resistance to vancomycin in lactic acid bacteria

6. Conclusions

\section{Introduction}

Gram-positive bacteria, such as the Enterococcus species, S. aureus and Clostridium difficile can be treated with vancomycin and similar glycopeptides, which are medications of last resort. Vancomycin's bactericidal effect was long hypothesized to be impervious to resistance as it binds to the bacterial cell

Correspondence to: Dr Samy Selim, Department of Clinical Laboratory Sciences, College of Applied Medical Sciences, Jouf University, Sakaka 72341, Kingdom of Saudi Arabia

E-mail: sabdulsalam@ju.edu.sa

Key words: mechanisms, resistance, vancomycin, gram-positive bacteria envelope rather than to a protein target, as is the case for other antibiotics. Vancomycin's therapeutic efficacy is threatened by two types of complex resistance mechanisms, each involving a multi-enzyme route, that have arisen and are becoming extensively more common in pathogenic species. Precursor degradation and substitution with DAla-D lac or D-Ala-D-Ser alternatives, which vancomycin has a poor affinity for, constitute the mechanisms of resistance. Vancomycin resistance has been studied extensively for $>30$ years, and significant progress has been made in the understanding of the molecular biology of the enzyme cascades involved $(1,2)$. The large size and high molecular weights of glycopeptides make them ineffective against gram-negative bacteria as they cannot penetrate past the outer membrane. Vancomycin is effective against all gram-negative bacteria, but nearly all gram-positive bacteria are vulnerable to glycopeptides (3). Inhibition of the bacterial cell wall by glycopeptides results in exposure of the fragile cytoplasmic membranes surrounding the bacterial cells, resulting in their bactericidal activity. There is an increase in osmotic pressure inside bacterium cells, causing them to quickly expand until they burst open (4). This review summarizes the mechanisms of gram-positive vancomycin resistance, which may be useful in the cure of illnesses caused by these pathogens.

\section{Mechanisms of vancomycin resistant Enterococci (VRE)}

vanA. Novel therapies for VRE are urgently required according to the World Health Organization (4). Vancomycin resistance is potent [Minimum Inhibitory Concentration (MIC) $64 \mu \mathrm{g} / \mathrm{ml}$, and resistance to teicoplanin is modest $(16-51 \mu \mathrm{g} / \mathrm{ml})$ in Enterococci. E. faecalis, E. faecium and E. casseliflavus are the most common sources of van A Enterococci. However, this type of resistance is also found, although more rarely, in E. avium, E. durans, E. gallinarum and E. raffinosus (5). A gene cluster on the transposon Tn1546 controls vanA resistance. Transposons are normally found on plasmids, although they can also be situated on bacterial chromosomes and vice versa (6). The resistance genes can be passed on to various Enterococci species (6) and even to other genera, such as Staphylococci, through conjugation $(6,7)$. It has been established that when the vanA cluster of genes is expressed, peptidoglycan precursors are synthesized that end in a depsipeptide termed D-Alanyl-D-lactate (D-Lac) rather than the usual D-Ala-D-Ala end. Vancomycin has a far lower affinity for D-Ala-D-Lac than it does for a regular 
dipeptide product (8-10). When the vanA protein is activated, it produces the depsipeptide D-Ala-D-Lac as a byproduct (11). To use the protein coded by van $\mathrm{H}$ in the above reaction, pyruvate must first be converted to D-lactate by reducing it with a D-hydroxy acid dehydrogenase enzyme (12). When it comes to the enzymes that break down D-Ala-D-Lac, the vanX protein is inactive. In order to reduce competitive pentapeptide synthesis, D-hydroxy acid dehydrogenase cleaves the D-Ala-Dla generated by conventional ligase activity $(13,14)$.

Additionally, the vanA cluster contains the regulatory genes vanS and vanR, which control the transcription of the aforementioned vanH, Van A and vanX genes, in addition to the crucial genes stated above. As the gene's protein product is a membrane-associated sensor protein that detects the presence of vancomycin via an unknown method, the gene was named vanS (15). A sensor protein that has been triggered by vancomycin signals the cytoplasmic vanR protein (the response regulator), which functions as a transcriptional activator and activates the promoter located between the vanS and vanH genes, to send signals. This then stimulates co-transcription of the chief glycopeptide resistance genes, vanH, VanA and xenopus tubulin (16).

Vancomycin resistance is formed by the united bustle of the three enzymes represented by the three primary resistance genes: vanH, vanA, and vanX, and cannot be produced by the vanA gene alone. VanR and vanS are important regulatory genes that govern the primary resistance genes (15). An additional two genes, vanY and vanZ, are carried by the transposon Tn1546 and are positioned downstream of the gene cluster vanA. These two genes can aid glycopeptide resistance, although they are not required for it (17). With regard to the vanY protein (also known as D,D-carboxypeptidase), the vanA cluster appears to be the key regulator. Transcriptional control of the vanA cluster has been shown to be complicated, as evidenced by the fact that the vanY protein is produced when vanA is present. vanY eliminates any normal membrane-bound peptidoglycan precursors that evade the action of the vanA gene cluster by cleaving the D-Ala terminus. As a result of vanY activity, glycopeptide antibiotic resistance is increased (17). The vanZ protein raises the MICs for teicoplanin, but not for vancomycin; however, the mechanism underlying this remains to be elucidated (18).

vanB. Vancomycin-resistant Enterococcal bacteria become vancomycin resistance at low levels of resistance (MIC $>8 \mu \mathrm{g} / \mathrm{ml}$ ), but are sensitive to teicoplanin. Only vancomycin, but not teicoplanin, induces vanB resistance, whereas the previously described vanA resistance is induced after treatment with either vancomycin or teicoplanin. Most often, the vanB phenotype is found in E. faecalis, although it can also be found in other Enterococcal species on rare occasions (8). The gene cluster controlling vanB resistance is the same as that controlling vanA resistance (vanB gene and related genes). Tn1547 is the transposon on which the vanB class gene cluster resides. While it can also be found on plasmids, this transposon is more commonly found in the bacteria's chromosomes (19). Some Enterococci (20) and other genera, such as $S$. bovis, carry the vanB type of vancomycin resistance gene through conjugation (21). This gene's protein product (vanB) is physically related to the vanA ligase but functions abnormally
(76\% amino acid homology). Using the pentapeptide ending in the depsipeptide D-Ala-D-Lac, vanB ligase promotes the formation of aberrant peptidoglycan precursors (22).

Genetic resistance genes of the vanB type, which correspond to vanA type, are classified as follows: vanRB, vanSB, vanHB and vanXB. However, unlike the van A class vanS protein, which does recognize teicoplanin and is responsible for inducing vanA resistance, the vanSB protein does not detect this glycopeptide. vanB-resistant bacteria lack the vanZ gene, which is only found in vanA-resistant bacteria (23). The vanB gene reveals DNA sequence variability, allowing the subtyping of the vanB gene into three subtypes: vanB-1, vanB-2 and vanB-3 (24,25). vanB-2 is the mst potent gene of the vanB family (26).

vanC. Unlike van A and vanB resistance, which can be acquired and transferred, the vanC type of resistance was found in E. gallinarum, E. casseliflavus, and E. flavescens as an innate constitutive feature (27). This type of resistance is characterized as resistant to vancomycin (MIC 8-32 $\mu \mathrm{g} / \mathrm{ml}$ ) but sensitivity to teicoplanin $(8 \mu \mathrm{g} / \mathrm{ml})$. Genes on the bacterial chromosome govern this sort of resistance, which cannot be passed from one individual to another $(27,28)$. The vanC gene's protein output is a dipeptide called D-Alanyl-D-Serine, which terminates in a pentapeptide called vanC. This change in the peptidoglycan production impairs vancomycin binding and abrogates its function (29). In vanC-resistant bacteria, different quantities of D-Ala-D-Ser are present in comparison to normal levels, which may explain the varying levels of vancomycin resistance $(30,31)$. E. gallinarum has a vanC-1 gene, E. casseliflavus has a vanC-2 gene and E. flavescens has a vanC-3 gene (19). Vancomycin resistance (MIC $>256 \mu \mathrm{g} / \mathrm{ml}$ ) and teicoplanin resistance (vanA genes have been found in several E. gallinarum and E. casseliflavus strains), and the number of genes conferring resistance was higher than expected (32).

vanD. In 1997, researchers discovered a novel gene which conferred vancomycin resistance which they named vanD. When tested against vancomycin and teicoplanin, the resistant strain of E. faecium showed a MIC of 64 and $4 \mu \mathrm{g} / \mathrm{ml}$, respectively (33). Further research showed that the vanD gene is a ligase gene that is distinct from, but comparable to, the van $\mathrm{A}$ and vanB genes, and the amino acid sequence of vanD showed $67 \%$ homology to the vanA genes. Enterococci cannot share the same vanD gene as it is situated on chromosomes (34). According to more recent research, vanD-type Enterococci resistant to vancomycin synthesize only peptidoglycan originators lacking the D-Ala-D-Lac, which confers constitutive resistance to vancomycin on those bacteria. The vanD gene encodes a ligase similar to the vanA and vanB types. Another gene belonging to the vanD cluster has been discovered that is analogous to the vanA-type genes. This gene, which encodes a dehydrogenase (vanHD), was termed vanHD. The dipeptidase (vanXD) gene has been termed vanXD (35).

vanE. E. faecalis BM4405, which is resistant to low concentrations of vancomycin (MIC $16 \mu \mathrm{g} / \mathrm{ml}$ ) but susceptible to teicoplanin (MIC $0.5 \mu \mathrm{g} / \mathrm{ml}$ ), possesses a more recently discovered vancomycin resistance gene named vanE (36). 
This novel type of resistance resembles that conferred by the intrinsic vanC type. A larger proportion of amino acid sequence similarity may be found in vanE $(55 \%)$ than in other van proteins (45\%) (36).

\section{Vancomycin resistance in Enterococci is conferred by several genes}

There has been evidence of both vancomycin resistance and dependency in some Enterococci strains of the vanA and vanB vancomycin resistance classes. The vancomycin-dependent Enterococci have been isolated by plating them on vancomycin-containing agar, such as that used for the separation of Campylobacter or Gonococci, from clinical samples that seemed to be culture negative (36). If these organisms stop producing D-Ala-D-Ala, one hypothesis is that they will be unable to continue grow without a new structure to replace it. It is only when vancomycin is present that most van $A$ and $\operatorname{van} B$ types of resistance occur, as vancomycin induces the creation of vanH dehydrogenase and of D-ala-D-lac-ligase (30). As long as vancomycin is present, bacteria may be unable to produce D-Ala-D-Ala, and even vanX destroy bacteria. This may explain why bacteria are unable to synthesize cell walls whilst D-Ala-D-Ala is present. As soon as the vancomycin is removed, the cell stops growing and replicating as it needs to either produce D-Ala or D-Ala D-Lac in order to continue growing and replicating $(36,30)$. If vancomycin independence is restored, it may be explained by a mutation leading to constitutive D-Ala-D-Lac production or via (re-)activation of the standard mechanism for synthesizing D-Ala-DAla $(36,37)$.

\section{Mechanisms of resistance to vancomycin in Staphylococci}

S. aureus strains, particularly methicillin-resistant strains, have been found to exhibit tolerance to vancomycin, according to early findings (38). Tolerance has been proposed as the reason for the failure of vancomycin treatment of infections caused by these bacteria despite the presence of MIC-detected susceptibility. Studies have also indicated that these resistant S. aureus strains increase mortality in individuals with bacteremia or endocarditis, despite vancomycin therapy $(38,39)$. Vancomycin-resistant $S$. aureus (VRSA) strains have been identified, and these bacteria have in-vitro MIC values $>4 \mu \mathrm{g} / \mathrm{ml}$. Vancomycin resistance has been observed in both S. aureus and coagulase-negative staphylococci (40). VRSA is more prevalent in Africa and Asia than in Europe and the United States, and vanA and SCCmec II were the most common genetic factors associated with VRSA (41).

The emergence of VRSA was particularly noticeable in institutions with a high prevalence of methicillin-resistant Staphylococci and a policy of indiscriminate glycopeptide administration. The extensive use of glycopeptides (vancomycin or teicoplanin) has permitted the selection of resistant strains from both $S$. aureus and coagulase negative Staphylococci, primarily from S. epidermidis, followed by $S$. hemolyticus, S. hominis and S. warneri (42-44). Additionally, glycopeptide-resistant mutants of $S$. aureus have been selected experimentally by gradually increasing the quantity of vancomycin present during in vitro development, supporting this theory (9). Furthermore, the discovery that vancomycin heteroresistance is a widespread occurrence among Staphylococci also supports this theory $(45,46)$. When vancomycin is used to treat bacterial infections, the presence of heteroresistance may allow for the selection of resistant strains in the body.

Due to the discovery of the vancomycin heteroresistance phenomena in Staphylococci, the procedures employed in the clinical microbiology laboratories to distinguish vancomycin resistance may need updating/adapting. The inoculum tested must be large enough for justification of the occurrence of the phenotype. Staphylococci vancomycin resistance has been shown to be reversible in the lab. S. aureus isolates with vancomycin resistance were repeatedly cultured on non-selective media in an effort to demonstrate this phenotypic reversal. Moreover, the vancomycin-resistant subpopulations were eliminated from several obtained strains. Reversion of vancomycin resistance phenotype may account for the difficulty in obtaining vancomycin-resistant clinical Staphylococci isolates from patients who do not respond to vancomycin therapy, as well as some of the challenges in identifying these isolates within the clinical microbiology laboratory (47). Primary testing of a vancomycin-resistant $S$. epidermidis strain was performed in a case study. A second test was accomplished on the isolate cultured to validate the first result, and the outcome was that the strain remained sensitive to vancomycin even after multiple tests. As it was unclear whether the initial primary test was flawed, researchers performed a second round of testing on the same patient (48). According to the data, there was no error in the primary susceptibility test, but there was a problem with an obscure phenotype (49). These findings may lay the groundwork for a novel approach to the clinical laboratory cultivation of Staphylococci. Vancomycin-resistant isolates can be maintained on the normal media as well as a medium containing low levels of the antibiotic, which may be preferable. The basic results of vancomycin susceptibility testing, not the results from subcultures, should be taken into account as well. The method by which Staphylococci develop resistance to vancomycin remains unknown at present, to the best of our knowledge. However, in vitro experiments and mouse skin transfers of the vanA gene cluster from Enterococci to $S$. aureus have been demonstrated in the laboratory. Clinical isolates of Staphylococci, which frequently co-colonize wound infection sites with Enterococci, may exhibit this route of genetic transmission (50). However, no investigation has shown the presence of any van gene clusters in clinical Staphylococci isolates, and hence a mechanism of this nature has not been observed (51).

Vancomycin-resistant Staphylococci in a liquid media form bacterial cell aggregates when exposed to vancomycin. Following this, the drug is no longer detected in the medium, and the bacterial aggregates break down into single cells with the same appearance as cells grown in an antibiotic-free environment. Electron microscopy examination of the bacterial aggregates revealed the development of substantial amounts of extracellular material with staining qualities comparable to those of the cell wall. Bioactive vancomycin may be extracted from cell wall material. These findings suggest that bacteria may sequester antibiotic molecules in a bound form on an additional cell wall substance, making them resistant to the activity of vancomycin (52-54). 
A total of 16 vancomycin-resistant $S$. aureus clinical isolates from 7 countries were tested in a drug-free medium. Vancomycin-susceptible strains (MIC $4 \mu \mathrm{g} / \mathrm{ml}$ ) were obtained after 10-84 days of these passages, but reversion to vancomycin resistance was achieved by a single step of vancomycin selection. All vancomycin-resistant strains had thicker cell walls that became thinner when vancomycin resistance was lost and thick again when vancomycin resistance was regained in a laboratory setting. $S$. aureus vancomycin resistance can develop under the selective pressure of long-term vancomycin use, but the resistance can be overcome by discontinuing the drug's use. It is possible, according to this research, that vancomycin resistance is caused by thickening of the bacterial cell wall, or some property associated with this (55).

Even though vancomycin-resistant Staphylococci thickening has been linked to complex reorganization of cell wall metabolism (10), with additional wall material displaying lower peptidoglycan cross-linking of the side chains of D-Ala (56). The complex reorganization of cell metabolism and lower peptidoglycan cross-linking prevents vancomycin from reaching the intracellular target molecules as it binds to these free termini outside the cell wall (54). In these bacteria, the thickening and metabolic reorganization of the cell wall are likely acquired through a genetic mutation $(38,39)$. In order to better understand how these strains develop vancomycin resistance, additional research is needed to improve our understanding of vancomycin resistance in these strains and highlight potential therapeutic options.

\section{Mechanisms of resistance to vancomycin in lactic acid bacteria}

The lactic acid bacteria Lactobacillus, Leuconostoc and Pediococcus are more likely to exhibit/attain vancomycin resistance. Human commensal lactic acid bacteria have been isolated from the digestive and genitourinary tracts of individuals, as well as from dairy products. Vancomycin is ineffective against lactic acid bacteria as these bacteria are genetically resistant to it, due to the weak affinity of vancomycin binding to their cell walls $(32,33)$. This weak binding was shown to be caused by the establishment of peptidoglycan precursors terminating in the depsipeptide D-Ala-D-Lac observed in Enterococci's vanA and vanB resistance classes to vancomycin, highlighting a potential direction for analysis of the peptidoglycans present in their cell walls. DNA probes for the vanA or vanB genes failed to hybridize with glycopeptide-resistant lactic acid bacteria. These findings suggested that these natively glycopeptide-resistant bacteria included a natural ligase that catalyzes the production of D-Ala-D-Lac instead of D-Ala-D-Ala, to which vancomycin binds with high affinities $(24,43)$.

\section{Conclusions}

There have been significant breakthroughs in our understanding of the molecular processes and genetics of vancomycin resistance in gram-positive bacteria. Despite this, it remains unknown where the resistance genes have originated from. A potential source of resistance for glycopeptide-producing organisms is the presence of genes encoding homologues of van A, vanH, vanR, vanS and vanX in these species' secondary metabolic products. For infections with multi-resistant strains of Staphylococci, Streptococci and Enterococci, glycopeptides, alone or in combination with another antibacterial, are typically the sole mode of treatment. Vancomycin-resistant gram-positive bacteria can become resistant to all antibiotics if they develop and spread significant levels of resistance to the drug. Therefore, knowledge of the molecular and structural vancomycin resistance pathways may highlight potential avenues for addressing this serious issue.

\section{Acknowledgements}

The author would sincerely like to thank the Jouf University, Kingdom of Saudi Arabia, for their unbounded support.

\section{Funding}

No funding was received.

\section{Availability of data and materials}

The datasets used and/or analyzed during the present study are available from the corresponding author on reasonable request.

\section{Authors' contributions}

SS wrote and revised the manuscript. The author has read and approved the final manuscript.

\section{Ethics approval and consent to participate}

Not applicable.

\section{Patient consent for publication}

Not applicable.

\section{Competing interests}

The author has declared that no competing interest exists.

\section{References}

1. Stogios PJ and Savchenko A: Molecular mechanisms of vancomycin resistance. Protein Sci 29: 654-669, 2020.

2. Barna JC and Williams DH: The structure and mode of action of glycopeptide antibiotics of the vancomycin group. Annu Rev Microbiol 38: 339-357, 1984.

3. Griffith RS: Vancomycin use - an historical review. J Antimicrob Chemother 14 (Suppl D): 1-5, 1984.

4. Mühlberg E, Umstätter F, Kleist C, Domhan C, Mier W and Uhl P: Renaissance of vancomycin: Approaches for breaking antibiotic resistance in multidrug-resistant bacteria. Can J Microbiol 66: $11-16,2020$

5. Reynolds PE: Structure, biochemistry and mechanism of action of glycopeptide antibiotics. Eur J Clin Microbiol Infect Dis 8: 943-950, 1989.

6. Morrison D, Woodford N and Cookson B: Enterococci as emerging pathogens of humans. J Appl Microbiol 83 (Suppl 1): 89S-99S, 1997.

7. Arthur M, Molinas C, Depardieu F and Courvalin P: Characterization of Tn1546, a Tn3-related transposon conferring glycopeptide resistance by synthesis of depsipeptide peptidoglycan precursors in Enterococcus faecium BM4147. J Bacteriol 175: 117-127, 1993. 
8. Brisson-Noël A, Dutka-Malen S, Molinas C, Leclercq R and Courvalin P: Cloning and heterospecific expression of the resistance determinant van A encoding high-level resistance to glycopeptides in Enterococcus faecium BM4147. Antimicrob Agents Chemother 34: 924-927, 1990.

9. Noble WC, Virani Z and Cree RGA: Co-transfer of vancomycin and other resistance genes from Enterococcus faecalis NCTC 12201 to Staphylococcus aureus. FEMS Microbiol Lett 72: 195-198, 1992.

10. Woodford N: Epidemiology of the genetic elements responsible for acquired glycopeptide resistance in enterococci. Microb Drug Resist 7: 229-236, 2001.

11. Bugg TDH, Wright GD, Dutka-Malen S, Arthur M, Courvalin P and Walsh CT: Molecular basis for vancomycin resistance in Enterococcus faecium BM4147: Biosynthesis of a depsipeptide peptidoglycan precursor by vancomycin resistance proteins VanH and VanA. Biochemistry 30: 10408-10415, 1991.

12. Pootoolal J, Neu J and Wright GD: Glycopeptide antibiotic resistance. Annu Rev Pharmacol Toxicol 42: 381-408, 2002.

13. Bugg TD, Dutka-Malen S, Arthur M, Courvalin P and Walsh CT: Identification of vancomycin resistance protein VanA as a D-alanine:D-alanine ligase of altered substrate specificity. Biochemistry 30: 2017-2021, 1991.

14. Arthur M, Molinas C, Dutka-Malen S and Courvalin P. Structural relationship between the vancomycin resistance protein VanH and 2-hydroxycarboxylic acid dehydrogenases. Gene 103: 133-134, 1991.

15. Reynolds PE, Depardieu F, Dutka-Malen S, Arthur M and Courvalin P: Glycopeptide resistance mediated by enterococcal transposon Tn1546 requires production of VanX for hydrolysis of D-alanyl-D-alanine. Mol Microbiol 13: 1065-1070, 1994.

16. Wu Z, Wright GD and Walsh CT: Overexpression, purification, and characterization of VanX, a D-, D-dipeptidase which is essential for vancomycin resistance in Enterococcus faecium BM4147. Biochemistry 34: 2455-2463, 1995.

17. Arthur M and Quintiliani R Jr: Regulation of VanA- and VanB-type glycopeptide resistance in enterococci. Antimicrob Agents Chemother 45: 375-381, 2001

18. Arthur M, Depardieu F, Gerbaud G, Galimand M, Leclercq R and Courvalin P: The VanS sensor negatively controls VanR-mediated transcriptional activation of glycopeptide resistance genes of Tn1546 and related elements in the absence of induction. J Bacteriol 179: 97-106, 1997.

19. Arthur M, Depardieu F, Molinas C, Reynolds $P$ and Courvalin $P$ : The vanZ gene of Tn1546 from Enterococcus faecium BM4147 confers resistance to teicoplanin. Gene 154: 87-92, 1995.

20. Arthur M, Molinas C and Courvalin P: Sequence of the vanY gene required for production of a vancomycin-inducible D,D-carboxypeptidase in Enterococcus faecium BM4147. Gene 120: 111-114, 1992.

21. Evers S, Sahm DF and Courvalin P: The VanB gene of Enterococcus faecalis V583 is structurally related to genes encoding D-Ala-D-Ala ligases and glycopeptide resistance proteins VanA and VanC. Gene 124: 143-144, 1993.

22. Hayden MK, Picken RN and Sahm DF: Heterogeneous expression of glycopeptide resistance in enterococci associated with transfer of vanB. Antimicrob Agents Chemother 41: 872-874, 1997.

23. Poyart C, Pierre C, Quesne G, Pron B, Berche P and Trieu-Cuot P: Emergence of vancomycin resistance in the genus Streptococcus: Characterization of a vanB transferable determinant in Streptococcus bovis. Antimicrob Agents Chemother 41: 24-29, 1997.

24. Baptista M, Depardieu F, Courvalin P and Arthur M: Specificity of induction of glycopeptide resistance genes in Enterococcus faecalis. Antimicrob Agents Chemother 40: 2291-2295, 1996.

25. Billot-Klein D, Gutmann L, Sablé S, Guittet E and van Heijenoort J: Modification of peptidoglycan precursors is a common feature of the low-level vancomycin-resistant VANB-type Enterococcus D366 and of the naturally glycopeptide-resistant species Lactobacillus casei, Pediococcus pentosaceus, Leuconostoc mesenteroides, and Enterococcus gallinarum. J Bacteriol 176: 2398-2405, 1994.

26. Boyle-Valva S, Berke SK, Lee JC and Daum RS: Reversion of the glycopeptide resistance phenotype in Staphylococcus aureus clinical isolates. Antimicob Agents Chemother 44: 272-277, 2000.

27. Cercenado E, García-Leoni ME, Díaz MD, Sánchez-Carrillo C, Catalán P, De Quirós JC and Bouza E: Emergence of teicoplanin-resistant coagulase-negative staphylococci. J Clin Microbiol 34: 1765-1768, 1996.

28. Clark NC, Teixeira LM, Facklam RR and Tenover FC: Detection and differentiation of vanC-1, vanC-2, and vanC-3 glycopeptide resistance genes in enterococci. J Clin Microbiol 36: 2294-2297, 1998.
29. Cui L, Ma X, Sato K, Okuma K, Tenover FC, Mamizuka EM, Gemmell CG, Kim MN, Ploy MC, El-Solh N, et al: Cell wall thickening is a common feature of vancomycin resistance in Staphylococcus aureus. J Clin Microbiol 41: 5-14, 2003.

30. Dahl KH, Simonsen GS, Olsvik O and Sundsfjord A: Heterogeneity in the vanB gene cluster of genomically diverse clinical strains of vancomycin-resistant enterococci. Antimicrob Agents Chemother 43: 1105-1110, 1999.

31. Dever LL, Smith SM, Handwerger S and Eng RH: Vancomycindependent Enterococcus faecium isolated from stool following oral vancomycin therapy. J Clin Microbiol 33: 2770-2773, 1995.

32. Dunne WM Jr, Qureshi H, Pervez H and Nafziger DA: Staphylococcus epidermidis with intermediate resistance to vancomycin: Elusive phenotype or laboratory artifact? Clin Infect Dis 33: 135-137, 2001

33. Dutka-Malen S, Blaimont B, Wauters G and Courvalin P: Emergence of high-level resistance to glycopeptides in Enterococcus gallinarum and Enterococcus casseliflavus. Antimicrob Agents Chemother 38: 1675-1677, 1994.

34. Dutka-Malen S, Leclercq R, Coutant V, Duval J and Courvalin P: Phenotypic and genotypic heterogeneity of glycopeptide resistance determinants in gram-positive bacteria. Antimicrob Agents Chemother 34: 1875-1879, 1990.

35. Eliopoulos GM: Vancomycin-resistant enterococci. Mechanism and clinical relevance. Infect Dis Clin North Am 11: 851-865, 1997.

36. Fahmy K and El-Hendi Y: Emergence of glycopeptide nonsusceptible coagulase negative staphylococci among renal failure patients undergoing peritoneal dialysis. Egypt J Med Microbiol 11: 349-356, 2002

37. Fines M,Perichon B, Reynolds P,Sahm DF and Courvalin P: VanE, a new type of acquired glycopeptide resistance in Enterococcus faecalis BM4405. Antimicrob Agents Chemother 43: 2161-2164, 1999.

38. Fridkin SK: Vancomycin-intermediate and -resistant Staphylococcus aureus: What the infectious disease specialist needs to know. Clin Infect Dis 32: 108-115, 2001

39. Geisel R, Schmitz FJ, Fluit AC and Labischinski H: Emergence, mechanism, and clinical implications of reduced glycopeptide susceptibility in Staphylococcus aureus. Eur J Clin Microbiol Infect Dis 20: 685-697, 2001.

40. Hiramatsu K: Vancomycin-resistant Staphylococcus aureus: A new model of antibiotic resistance. Lancet Infect Dis 1: 147-155, 2001.

41. Asadpour L and Ghazanfari N: Detection of vancomycin nonsusceptible strains in clinical isolates of Staphylococcus aureus in northern Iran. Int Microbiol 22: 411-417, 2019.

42. Wu Q, Sabokroo N, Wang Y, Hashemian M, Karamollahi S and Kouhsari E: Systematic review and meta-analysis of the epidemiology of vancomycin-resistance Staphylococcus aureus isolates. Antimicrob Resist Infect Control 10: 101, 2021.

43. Murray BE: Vancomycin-resistant enterococcal infections. N Engl J Med 342: 710-721, 2000.

44. Nicas TI, Cole CT, Preston DA, Schabel AA and Nagarajan R: Activity of glycopeptides against vancomycin-resistant grampositive bacteria. Antimicrob Agents Chemother 33: 1477-1481, 1989

45. OstrowskyBE,ClarkNC, Thauvin-EliopoulosC, Venkataraman L, Samore MH, Tenover FC, Eliopoulos GM, Moellering RC Jr and Gold HS: A cluster of VanD vancomycin-resistant Enterococcus faecium: Molecular characterization and clinical epidemiology. J Infect Dis 180: 1177-1185, 1999.

46. Perichon B, Reynolds P and Courvalin P: VanD-type glycopeptide-resistant Enterococcus faecium BM4339. Antimicrob Agents Chemother 41: 2016-2018, 1997.

47. Rajashekaraiah KR, Rice T, Rao VS, Marsh D, Ramakrishna B and Kallick CA: Clinical significance of tolerant strains of Staphylococcus aureus in patients with endocarditis. Ann Intern Med 93: 796-801, 1980.

48. Reynolds PE, Snaith HA, Maguire AJ, Dutka-Malen S and Courvalin P: Analysis of peptidoglycan precursors in vancomycin-resistantEnterococcusgallinarumBM4174.Biochem J 301: 5-8, 1994

49. Rodriguez-Cerrato V, McCoig CC, Saavedra J, Barton T, Michelow IC, Hardy RD, Bowlware K, Iglehart J, Katz K and McCracken GH Jr: Garenoxacin (BMS-284756) and moxifloxacin in experimental meningitis caused by vancomycin-tolerant pneumococci. Antimicrob Agents Chemother 47: 211-215, 2003.

50. Rybak MJ and Akins RL: Emergence of methicillin-resistant Staphylococcus aureus with intermediate glycopeptide resistance: Clinical significance and treatment options. Drugs 61: $1-7,2001$ 
51. Shariati A, Dadashi M, Moghadam MT, van Belkum A Yaslianifard S and Dar ban Sarokhalil DJ: Global prevalence and distribution of vancomycin resistant, vancomycin intermediate and heterogeneously vancomycin intermediate Staphylococcus aureus clinical isolates: a systematic review and meta analysis. Sci Rep 10: 1-16, 2020.

52. Sieradzki K, Villari P and Tomasz A: Decreased susceptibilities to teicoplanin and vancomycin among coagulase-negative methicillin-resistant clinical isolates of staphylococci. Antimicrob Agents Chemother 42: 100-107, 1998.

53. Cong Y, Yang S and Rao X: Vancomycin resistant Staphylococcus aureus infections: A review of case updating and clinical features. J Adv Res 21: 169-176, 2019.
54. Walsh TR and Howe RA: The prevalence and mechanisms of vancomycin resistance in Staphylococcus aureus. Annu Rev Microbiol 56: 657-675, 2002.

55. Wong SSY, Ho PL, Woo PCY and Yuen KY: Bacteremia caused by staphylococci with inducible vancomycin heteroresistance. Clin Infect Dis 29: 760-767, 1999.

56. Tacconelli E, Tumbarello M, Donati KG, Bettio M, Spanu T, Leone F, Sechi LA, Zanetti S, Fadda G and Cauda R: Glycopeptide resistance among coagulase-negative staphylococci that cause bacteremia: Epidemiological and clinical findings from a case-control study. Clin Infect Dis 33: 1628-1635, 2001. 